\title{
In vivo detection of dysregulated choline metabolism in paclitaxel-resistant ovarian cancers with proton magnetic resonance spectroscopy
}

\author{
Jing Lu' , Ying Li ${ }^{1}$, Yong Ai Li ${ }^{1}$, Li Wang ${ }^{2}$, An Rong Zeng ${ }^{1}$, Xiao Liang Ma and Jin Wei Qiang ${ }^{1 *}$ (D)
}

\begin{abstract}
Background: Chemoresistance gradually develops during treatment of epithelial ovarian cancer (EOC). Metabolic alterations, especially in vivo easily detectable metabolites in paclitaxel (PTX)-resistant EOC remain unclear.

Methods: Xenograft models of the PTX-sensitive and PTX-resistant EOCs were built. Using a combination of in vivo proton-magnetic resonance spectroscopy ( $\left.{ }^{1} \mathrm{H}-\mathrm{MRS}\right)$, metabolomics and proteomics, we investigated the in vivo metabolites and dysregulated metabolic pathways in the PTX-resistant EOC. Furthermore, we analyzed the RNA expression to validate the key enzymes in the dysregulated metabolic pathway.
\end{abstract}

Results: On in vivo ${ }^{1} \mathrm{H}-\mathrm{MRS}$, the ratio of (glycerophosphocholine + phosphocholine) to (creatine + phosphocreatine) $((\mathrm{GPC}+\mathrm{PC})$ to $(\mathrm{Cr}+\mathrm{PCr}))($ i.e. $\mathrm{Cho} / \mathrm{Cr})$ in the PTX-resistant tumors $(1.64[0.69,4.18])$ was significantly higher than that in the PTX-sensitive tumors $(0.33[0.10,1.13])(P=0.04)$. Forty-five ex vivo metabolites were identified to be significantly different between the PTX-sensitive and PTX-resistant tumors, with the majority involved of lipids and lipid-like molecules. Spearman's correlation coefficient analysis indicated in vivo and ex vivo metabolic characteristics were highly consistent, exhibiting the highest positive correlation between in vivo GPC + PC and ex vivo GPC $(r=0.885, P<0.001)$. These metabolic data suggested that abnormal choline concentrations were the results from the dysregulated glycerophospholipid metabolism, especially choline metabolism. The proteomics data indicated that the expressions of key enzymes glycerophosphocholine phosphodiesterase 1 (GPCPD1) and glycerophosphodiester phosphodiesterase 1 (GDE1) were significantly lower in the PTX-resistant tumors compared to the PTX-sensitive tumors (both $P<0.01$ ). Decreased expressions of GPCPD1 and GDE1 in choline metabolism led to an increased GPC levels in the PTX-resistant EOCs, which was observed as an elevated total choline (tCho) on in vivo ${ }^{1} \mathrm{H}-\mathrm{MRS}$.

Conclusions: These findings suggested that dysregulated choline metabolism was associated with PTX-resistance in EOCs and the elevated tCho on in vivo ${ }^{1} \mathrm{H}$-MRS could be as an indicator for the PTX-resistance in EOCs.

Keywords: Ovarian cancer, Drug resistance, Proton magnetic resonance spectroscopy, Metabolomics, Choline metabolism

*Correspondence: dr.jinweiqiang@163.com

${ }^{1}$ Department of Radiology, Jinshan Hospital, Fudan University, 1508 Longhang Road, Shanghai 201508, People's Republic of China

Full list of author information is available at the end of the article

\section{Background}

Epithelial ovarian cancer (EOC) is the most lethal gynecological malignancy and is the seventh most commonly diagnosed cancer among women worldwide, with $75 \%$ of patients diagnosed in the advanced stage and $46 \%$ original author(s) and the source, provide a link to the Creative Commons licence, and indicate if changes were made. The images or other third party material in this article are included in the article's Creative Commons licence, unless indicated otherwise in a credit line to the material. If material is not included in the article's Creative Commons licence and your intended use is not permitted by statutory regulation or exceeds the permitted use, you will need to obtain permission directly from the copyright holder. To view a copy of this licence, visit http://creativecommons.org/licenses/by/4.0/. The Creative Commons Public Domain Dedication waiver (http://creativeco mmons.org/publicdomain/zero/1.0/) applies to the data made available in this article, unless otherwise stated in a credit line to the data. 
exhibiting a 5-year survival rate after diagnosis [1]. The current standard treatment approach comprises cytoreductive surgery followed by combined chemotherapy with platinum and paclitaxel (PTX) [2]. The majority of the patients have an initial response to chemotherapy, e.g., cisplatin and PTX [3]. However, PTX resistance gradually develops during treatment, leading to therapeutic failure [4]. More than $75 \%$ of patients are likely to suffer tumor recurrence due to chemoresistance [2].

Metabolomics analysis can provide a real-world assessment of cancer cell physiology [5, 6]. A close relationship between deregulated metabolic reprogramming and drug resistance in cancer therapy has been reported [7-10]. Targeting metabolism might represent a potential option to overcome drug resistance [11]. Therefore, a better understanding of the adaptive tumor phenotype following treatment resistance and exploring the clinically applicable biomarkers of chemoresistance, are required to retrieve the chemosensitivity in EOC patients $[3,11]$. However, metabolic alterations, especially easily detectable metabolites, in PTX-resistant EOC remain unclear.

Metabolic changes have most commonly been studied using magnetic resonance spectroscopy (MRS) [12], which is a non-invasive functional imaging method on magnetic resonance imaging (MRI). A great advantage of MRS is the ability to detect intrinsic metabolic changes without the administration of an extrinsic marker [13]. Compared to benign ovarian tumors, an increased total choline ( $\mathrm{tCho}$ ) on proton $\left({ }^{1} \mathrm{H}\right)$-MRS has been considered to be a characteristic manifestation of EOC [14-17]. ${ }^{1} \mathrm{H}$ MRS studies report alterations in the spectral profile in the region of 3.20 to $3.24 \mathrm{ppm}$, which are indicative of tCho levels including glycerophosphocholine (GPC), phosphocholine (PC), and free Cho [12]. Moreover, metabolic changes have been reported after chemotherapy in malignant tumors, such as breast cancer and glioma, suggesting that these metabolic changes can be used as indicators of therapeutic response [18-22]. However, ${ }^{1} \mathrm{H}$ MRS studies concerning chemoresistance in EOC have not been performed.

In this study, we used a combination of in vivo ${ }^{1} \mathrm{H}-\mathrm{MRS}$, metabolomics and proteomics to perform an unbiased characterisation of tumors from PTX-resistant xenograft models (Fig. 1). We show that abnormal choline metabolism leads to PTX resistance in EOC and this can be accurately demonstrated by in vivo ${ }^{1} \mathrm{H}$-MRS. In addition, we identify glycerophosphocholine phosphodiesterase 1 (GPCPD1) and glycerophosphodiester phosphodiesterase 1 (GDE1) as key enzymes associated with PTX resistance, representing a potential therapeutic target.

\section{Methods}

Cell culture

All cells were cultured in RPMI 1640 medium (Sigma Aldrich, St Louis, MI, USA) with 10\% fetal bovine serum (FBS, Gibco, Thermo Fisher Scientific, Waltham, MA, USA) and maintained at $37{ }^{\circ} \mathrm{C}$ under $5 \% \mathrm{CO}_{2}$. PTXresistant OVCAR-3 (OV_PTX) cells were generated in the laboratory and kindly provided by Dr. GX Xu (Fudan University, China) [23]. OV_PTX cells were derived from parental OVCAR-3 (OV) cells by treating cells with the PTX (Sichuan Taiji Pharm, China) regimen through a gradually increasing PTX dose in RPMI 1640 medium with $10 \%$ FBS.

\section{Proliferation assay}

Cell proliferation was measured by Cell Counting Kit-8 (CCK-8, Dojindo Molecular Technologies Inc., Shanghai, China). OV and OV_PTX cells were plated in 96-well plates with a density of $5 \times 10^{3}$ cells per well and incubated for $24 \mathrm{~h}$. PTX was added with increasing concentrations from $0.001-10 \mu \mathrm{mol} / \mathrm{mL}$ to cells, which were then incubated for $48 \mathrm{~h}$. The cells were then incubated with $10 \mu \mathrm{L}$ of CCK- 8 per well for $1 \mathrm{~h}$ at $37{ }^{\circ} \mathrm{C}$. Absorbance was measured at a wavelength of $450 \mathrm{~nm}$ using a Bio-Tek ELX808IU absorbance microplate reader (BioTek Instruments Inc., USA).

\section{Xenograft models}

Female BALB/c nude mice (Jiesijie Laboratory Animal Company, Shanghai, China; age, 4-5 weeks; weight, 12-15 g) were used under approved animal care. In vivo experiments were performed in accordance with the guidelines formulated by the Ethics Review Committee of China Animal Experimental System and were approved by the ethics committee of Shanghai Municipal Public Health Clinical Center (No. 2020-A019-01). Twenty nude mice were randomly divided into the $\mathrm{OV}$ group and $\mathrm{OV}_{-}$ PTX group ( $\mathrm{n}=10$ in each group). Briefly, $5 \times 10^{6} \mathrm{OV}$ or OV_PTX cells/mouse were suspended in serum-free medium, and $0.1 \mathrm{~mL}$ of the cell suspension was injected subcutaneously in the right anterior limb. Mice were monitored daily and weighted every two days. Tumor size was measured with a caliper every two days for the greatest longitudinal diameter (length) and greatest transverse diameter (width). Volume was calculated using the modified elliptical formula (length $\times$ width $\left.^{2}\right) / 2$ [24]. Tumors were allowed to grow for 19 days after injection until a diameter of approximately $1.0 \mathrm{~cm}$ was measured, which were suitable for in vivo ${ }^{1} \mathrm{H}$-MRS. One mouse in the $\mathrm{OV}_{-}$ PTX group died before imaging. 


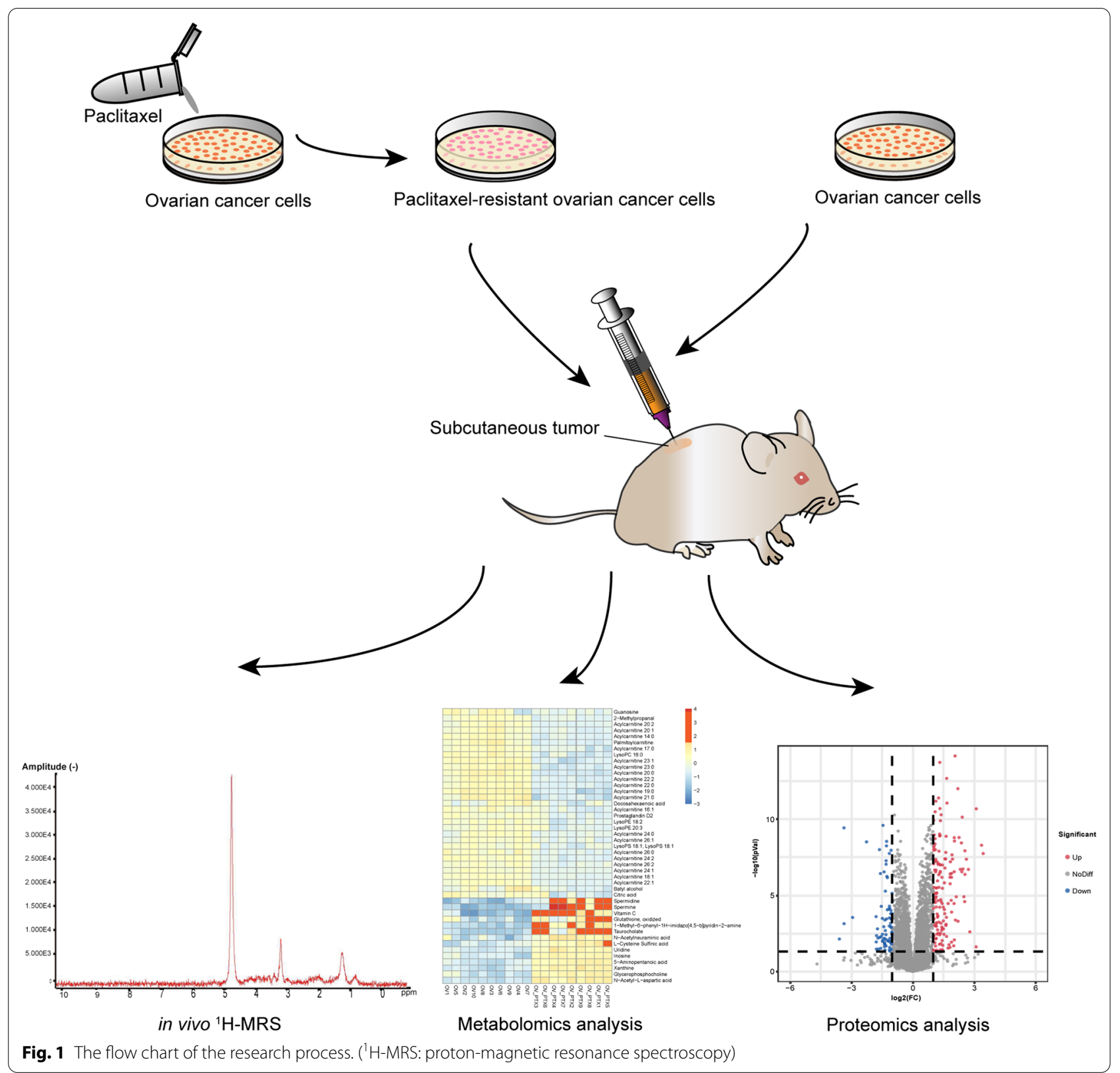

\section{In vivo ${ }^{1} \mathrm{H}-\mathrm{MRS}$}

Mice with OV or OV_PTX tumors were anesthetized with isoflurane $(1.5-2 \%)$ in oxygen $(1 \mathrm{~L} / \mathrm{min})$ and imaged in the prone position in a 7.0 T Biospec smallanimal MRI scanner (Bruker Corporation, Billerica, MA, USA). The imaging protocol included the following sequences:

1. T2-weighted rapid acquisition relaxation enhance: time of echo, $35 \mathrm{~ms}$; time of repetition, $2500 \mathrm{~ms}$; slice thickness, $0.4 \mathrm{~mm}$; field of view, $20 \times 20 \mathrm{~mm}$; matrix, $256 \times 256$; and number of averaged scans, 8 .
2. Single voxel point-resolved spectroscopy ${ }^{1} \mathrm{H}$-MRS: time of echo, $16 \mathrm{~ms}$; time of repetition, $2500 \mathrm{~ms}$; voxel size, $1.5 \times 1.5 \times 1.5 \mathrm{~mm}$; number of averaged scans, 128; and scan time, 5 min $20 \mathrm{~s}$.

The tumors were scanned on the transverse, coronal and sagittal planes using the T2 rapid acquisition relaxation enhance sequence for the three-dimensional positioning of ${ }^{1} \mathrm{H}$-MRS. Metabolite spectral fitting was performed using LCModel Version 6.3-0I, with a basis set provided by the LCModel software for a 7.0 T Bruker MRI scanner with time of echo $=16 \mathrm{~ms}$ [25]. Relative 
metabolite concentrations and their uncertainties were estimated by fitting the spectrum to a linear combination of basis spectra of each individual metabolite. The unsuppressed water spectrum was used to normalize the initial fit to generate a first estimate of metabolite concentration by scaling the relative areas and chemical shifts across the two sets of spectra. The spectral range for the analysis was set to $0.2-4.0 \mathrm{ppm}$ to contain most peaks of interest: alanine (Ala), aspartate (Asp), creatine $(\mathrm{Cr})$, phosphocreatine (PCr), glycerophosphocholine (GPC), phosphocholine (PC), inositol (Ins), lactate (Lac), taurine (Tau), N-acetylaspartate (NAA), N-acetylaspartylglutamate (NAAG), macromolecules (MM) 09, lipid (Lip) 09, Lip 13a, Lip 13b, MM12, MM14, MM20, Lip 20. The numbers after $\mathrm{MM}$ and Lip indicated the approximate chemical shift in ppm of the peaks; e.g., MM 14 for the macromolecule peak near $1.4 \mathrm{ppm}$. Only metabolite concentrations quantified with Cramèr-Rao lower bounds below $20 \%$ on average were included in further analysis [26]. With software (Fire Voxel, CAI2R, New York University, NY, USA), the region of interest was manually delineated slice-by-slice along the contour of the tumor on the transverse T2-weighted images (T2WIs) (M.X.L. and L.J., with 5 and 7 years of experience in gynecological imaging, respectively). Then, the volume of interest was postprocessed automatically for tumor anatomic measurement.

\section{Sample collection and histopathology}

In order to acquire a true map of in vivo metabolism, tumors were collected as soon as possible after MRI scanning with mice euthanized by excess $\mathrm{CO}_{2}$ exposure. Tumor samples were divided into multiple parts. One part was fixed with $4 \%$ paraformaldehyde and then embedded in paraffin and stained using hematoxylin and eosin for histological feature analysis. The other parts of tumor samples were flash-frozen in liquid nitrogen and were used for metabolomics analysis, proteomics analysis and quantitative RT-PCR, respectively.

\section{Metabolomics analysis}

Tumor samples $(100 \mathrm{mg})$ were extracted for analysis using by liquid chromatography-mass spectrometry (LC-MS). Pooled quality control samples were also prepared by combining the same volume of each sample and repeatedly injected during the assay to monitor instrumental stability and avoid systematic bias. The acquired LC-MS data was pretreated using XCMS software. Features with $<50 \%$ of quality control samples or $80 \%$ of test samples were removed, and values for missing peaks were extrapolated with the k-nearest neighbor algorithm to further improve the data quality. The group datasets were normalized before analysis. The detailed parameters were described in Additional file 1: Supplementary of metabolomics analysis method. Statistical analysis included principal component analysis (PCA), orthogonal partial least squares discriminant analysis (OPLS-DA), Student's $t$-test and fold change analysis. The $P$ value obtained by Student's $t$-test was then adjusted for multiple tests using a false discovery rate-reducing process (Benjamini-Hochberg) and was used to determine differential metabolites. The following criteria were used to screen the differential metabolites: variable importance in projection (VIP) sores $\geq 1, P$ value $<0.05$, and fold change $\geq 2$ or $\leq 0.5$. Pathway enrichment analysis of differential metabolites was performed using the Kyoto Encyclopedia of Genes and Genomes and MetaboAnalyst 3.0 (Montre al, QC, Canada) databases. Bioinformatic analysis was performed using the OmicStudio tools at https://www.omics tudio.cn/tool.

\section{Proteomics analysis}

Samples were lysed in sodium dodecyl sulfate buffer and homogenized. Proteins were digested overnight by trypsin (Promega, Madison, WI, USA), and the resulted peptides were collected as a filtrate. Pooled peptides from all samples were fractionated by reversed-phase chromatography using an Agilent 1260 infinity II HPLC (SCIEX, Framingham, MA, USA). The detailed parameters were described in Additional file 2: Supplementary of proteomics analysis method. Raw data of data-independent acquisition were processed and analyzed by Spectronaut 14.6 (Biognosys AG, Switzerland) with default settings. Data extraction was determined by Spectronaut X based on extensive mass calibration. Precursors which passed the filters were used for quantification.The average top 3 filtered peptides that passed the 1\% Q- value cutoff (false discovery rate) were used to calculate the major group quantities. Significantly enriched proteins were selected using Student's $t$-test.

\section{Quantitative RT-PCR}

Total RNA was extracted from tumors using Trizol reagent and reverse-transcribed into cDNA with $500 \mathrm{ng}$ of total RNA using the PrimeScript RT reagent kit (Thermo Fisher Scientific, Waltham, MA, USA) according to the manufacturer's instructions. Gene expression assays were performed on an ABI StepOnePlus Real-Time PCR instrument (Thermo Fisher Scientific, Waltham, MA, USA). The following custom designed primers were purchased from GENEWIZ Inc. (Suzhou, China): GPCPD1, GTTTTTGCGATATGTGGAAGCTG (forward) and AGCGATACTGAACTGATACTCCT (reverse); GDE1, GACTGGGCGATTGTGTGATTT (forward) and AGG GTAGGGATCTTTTCATCAGG (reverse); and $\beta$-Actin, 
GCCGTGGTGGTGAAGCTGT (forward) and ACC CACACTGTGCCCATCTA (reverse).

\section{Statistical analysis}

Continuous variables with normal distribution were presented as the mean \pm standard deviation; nonnormally distributed variables were presented as the median (interquartile range). All statistical analyses were performed with SPSS (version 23.0, SPSS, Inc., Chicago, IL, USA). The data were analyzed using Student's $t$-test or Mann-Whitney U test. Receiver operating characteristic curve analysis (MedCalc Software, Mariakerke, Belgium) was used to assess the diagnostic performance and determine a cutoff value for the significant metabolites from ${ }^{1} \mathrm{H}$-MRS to differentiate the PTX-sensitive and PTX-resistant tumors. The correlation of metabolites between in vivo ${ }^{1} \mathrm{H}$-MRS and ex vivo metabolomics analysis and the correlations between protein expressions and metabolite levels were analyzed using Spearman correlation tests. Differences with a $P<0.05$ were considered statistically significant.

\section{Results}

In vivo metabolic profile of PTX-resistant ovarian cancer

We performed the cell proliferation assay to confirm the PTX resistance, with a resistance index of $484.3 \pm 166.8$ (Fig. 2A). Cell atypia was more obvious in the OV_PTX tumor than that in OV tumor (Fig. 2B). Xenograft tumors were significantly larger in the OV group $(1028.5 \pm 370.5$ $\left.\mathrm{mm}^{3}\right)$ than in the OV_PTX group $\left(337.2 \pm 224.0 \mathrm{~mm}^{3}\right)$ $(P<0.001)$.

Eight mice from each group were successfully imaged. A total of 13 metabolites (Cramèr-Rao lower bounds $<20 \%$ ) were analysed, but no significant differences in frequencies of metabolite peaks were observed between the OV and OV_PTX tumors (Table 1; Fig. 2C). Thereinto, PC was very difficult to resolve from GPC, as was PCr from Cr. The sum of GPC + PC was much more

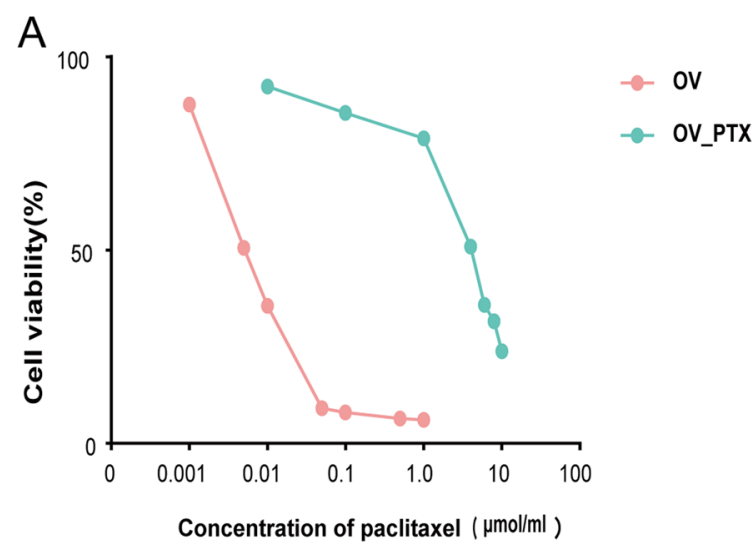

C

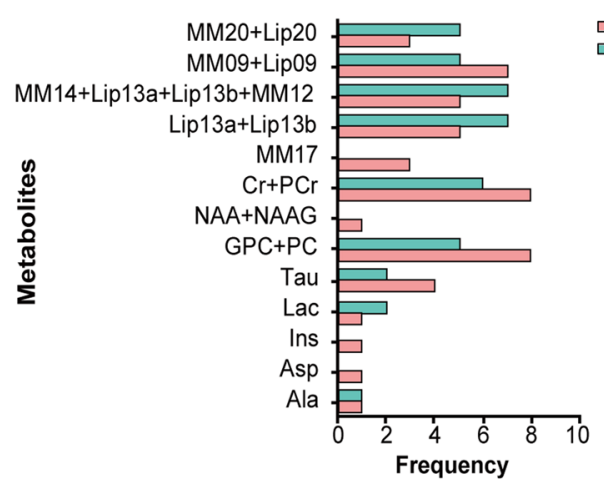

므을
B
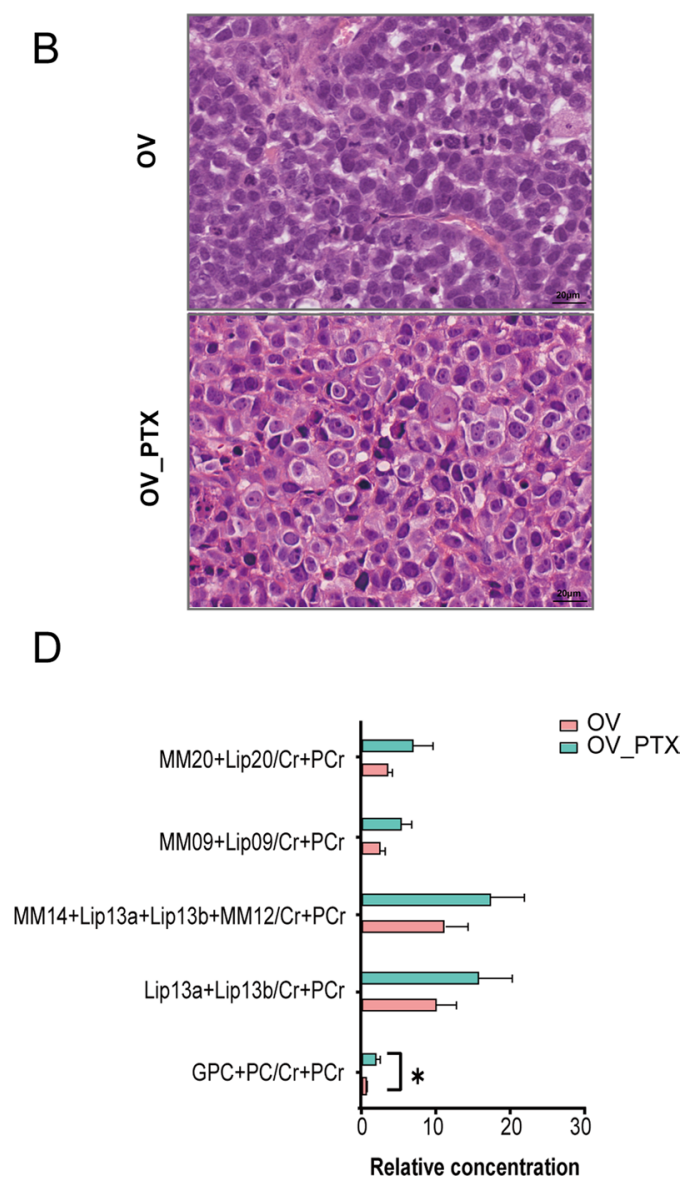

Fig. 2 In vivo metabolic profile of the PTX-sensitive and PTX-resistant tumors. Cell proliferation of OV and OV_PTX cells treated for $48 \mathrm{~h}$ with increasing concentrations of PTX (A). Hematoxylin and eosin staining of subcutaneous tumors showing the more obvious cell atypia and nuclear mitosis in the OV_PTX tumor cells $(400 \times$ magnification, scale bars $=20 \mu \mathrm{m})(\mathbf{B})$. The frequencies and relative concentrations of metabolite peaks in the OV and OV_PTX groups on in vivo ${ }^{1} \mathrm{H}-\mathrm{MRS}(\mathbf{C}, \mathbf{D}) .{ }^{*}, P<0.05$. PTX paclitaxel, OV: OVCAR-3 
Table 1 Metabolites detected in the OV and OV_PTX groups using ${ }^{1} \mathrm{H}-\mathrm{MRS}$

\begin{tabular}{|c|c|c|c|}
\hline Metabolites and ratios & $O V(n=8)$ & OV_PTX $(n=8)$ & $P$ value \\
\hline \multicolumn{4}{|l|}{ Metabolite peaks ${ }^{\mathrm{a}}$} \\
\hline Ala & 1 & 1 & - \\
\hline Asp & 1 & 0 & - \\
\hline Ins & 1 & 0 & - \\
\hline Lac & 1 & 2 & - \\
\hline Tau & 4 & 2 & 0.61 \\
\hline$G P C+P C$ & 8 & 5 & 0.20 \\
\hline$N A A+N A A G$ & 1 & 0 & - \\
\hline $\mathrm{Cr}+\mathrm{PCr}$ & 8 & 6 & 0.47 \\
\hline MM17 & 3 & 0 & - \\
\hline Lip13a + Lip13b & 5 & 7 & 0.57 \\
\hline MM14 + Lip13a + Lip13b + MM12 & 5 & 7 & 0.57 \\
\hline MM09 + Lip09 & 7 & 5 & 0.57 \\
\hline MM20 + Lip20 & 3 & 5 & 0.62 \\
\hline \multicolumn{4}{|c|}{ Metabolite concentration ratios $(/ \mathrm{Cr}+\mathrm{PCr})^{\mathrm{b}}$} \\
\hline $\mathrm{GPC}+\mathrm{PC}$ & $0.33(0.10,1.13)$ & $1.64(0.69,4.18)$ & 0.04 \\
\hline Lip13a + Lip13b & $11.48(5.88,18.28)$ & $15.15(9.96,31.86)$ & 0.43 \\
\hline MM14 + Lip13a + Lip13b + MM12 & $12.95(6.22,20.42)$ & $17.14(12.15,32.56)$ & 0.43 \\
\hline MM09+Lip09 & $3.10(0.84,3.58)$ & $4.65(3.40,9.92)$ & 0.09 \\
\hline MM20 + Lip20 & $3.28(3.13,5.56)$ & $4.19(2.75,15.25)$ & 0.46 \\
\hline
\end{tabular}

${ }_{1}^{1}$ H-MRS proton-magnetic resonance spectroscopy, OV ovarian cancer, OV_PTX paclitaxel-resistant ovarian cancer, Ala alanine, Asp aspartate, Ins inositol, Lac lactate, Tau taurine, $G P C+P C$ glycerophosphocholine + phosphocholine, $N A A+N A A G \mathrm{~N}$-acetylaspartate $+\mathrm{N}$-acetylaspartatglutamate, $C r+P C r$ creatine + phosphocreatine, Lip lipids, MM macromolecules, the numbers after MM and Lip indicate the approximate chemical shift in ppm of the peaks: e.g., MM17: macromolecule peak near 1.7 ppm

${ }^{\text {a }}$ Chi-square test

${ }^{\mathrm{b}}$ Mann-Whitney $\mathrm{U}$ test

accurate than the individual concentrations. Usually, the units of the absolute concentrations are unknown, and only concentration ratios are meaningful. LCModel metabolite ratios relative to $\mathrm{Cr}+\mathrm{PCr}$ were used for our analysis (as outlined in detail in the LCModel manual). The metabolite ratios and their differential levels in the OV and OV_PTX tumors are shown in Table 1 and Fig. 2D. The ratio of $(\mathrm{GPC}+\mathrm{PC})$ to $(\mathrm{Cr}+\mathrm{PCr})$ (i.e. Cho/ $\mathrm{Cr})$ in the OV_PTX group $(1.64[0.69,4.18])$ was significantly higher than that in the OV group $(0.33[0.10$, 1.13]) $(P=0.04)$. The metabolites of interest are displayed in Fig. $3 \mathrm{~A}$ and B. For differentiating the two groups of tumors, the optimal cutoff value of the $(\mathrm{GPC}+\mathrm{PC}) /$ $(\mathrm{Cr}+\mathrm{PCr})$ ratio was 1.216 , which yielded the sensitivity, specificity, and area under the curve (AUC) values of $100 \%, 80 \%$, and 0.85 (95\% confidence interval, 0.550 0.982), respectively (Fig. 3C). No significant differences in other 4 metabolite ratios were found between the OV and OV_PTX tumors, although higher ratios were detected in the OV_PTX tumors.

\section{Ex vivo metabolic profile of PTX-resistant ovarian cancer}

To decipher the mechanisms of in vivo metabolic imaging alterations associated with treatment resistance, we characterized the metabolome of the OV and OV_PTX tumors from xenograft models. As shown in the PCA plot, the metabolites of the OV group and the OV_PTX group were well separated (Fig. 4A; Additional file 3:

(See figure on next page.)

Fig. 3 Samples of MR spectra and the diagnostic efficiency of in vivo ${ }^{1} \mathrm{H}-\mathrm{MRS}$. ${ }^{1} \mathrm{H}-\mathrm{MRS}$ localization of the volume of interest and spectra acquired from the OV tumor (A) and OV_PTX tumor (B), along with fits and residuals of the fits resulting from LCModel quantification: $\mathrm{Cr}+\mathrm{PCr}(3.03 \mathrm{ppm})$, GPC+PC (3.21 ppm), MM14 + Lip 13a + Lip 13b + MM12 (1.2-1.4 ppm), MM 09+ Lip 09 (0.9 ppm), MM 20 + Lip 20 (2.0 ppm). The (GPC+ PC)/ $(\mathrm{Cr}+\mathrm{PCr})$ ratio was 1.18 for the OV tumor and 5.45 for the OV_PTX tumor. Receiver operating characteristic curve analysis of in vivo differential metabolite (GPC + PC) for differentiating the OV from OV_PTX groups (C). GPC glycerophosphocholine, $P C$ phosphocholine, $\mathrm{Cr}$ creatine, $P C r$ phosphocreatine, Lip lipids, MM macromolecules 
A

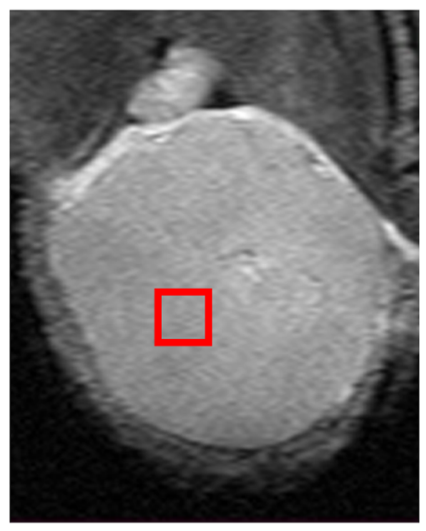

\section{$\stackrel{m}{\circ}$}

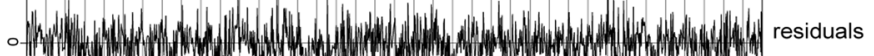

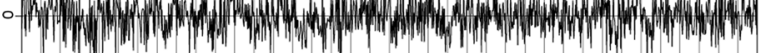

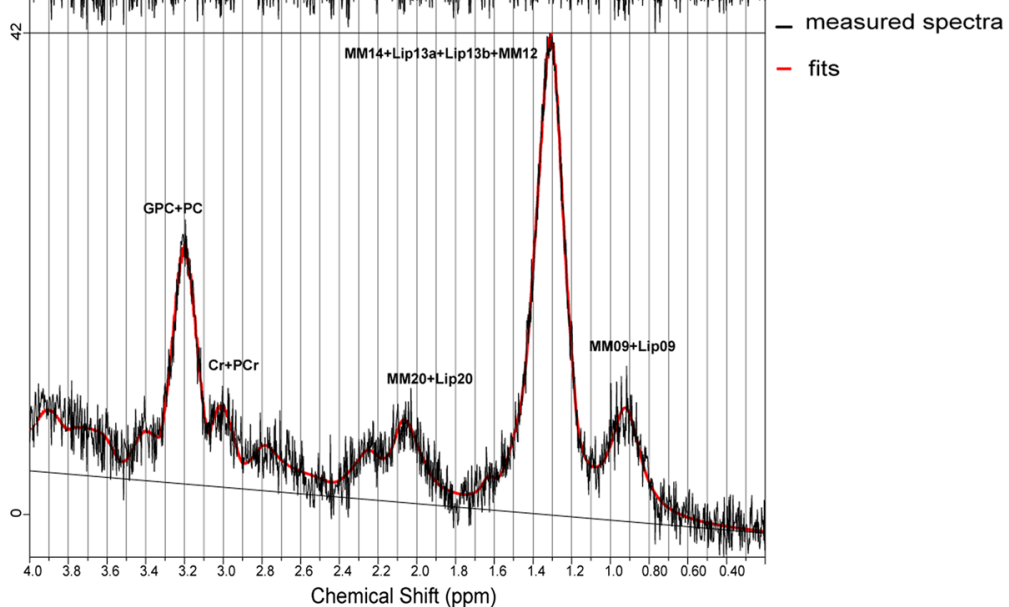

B
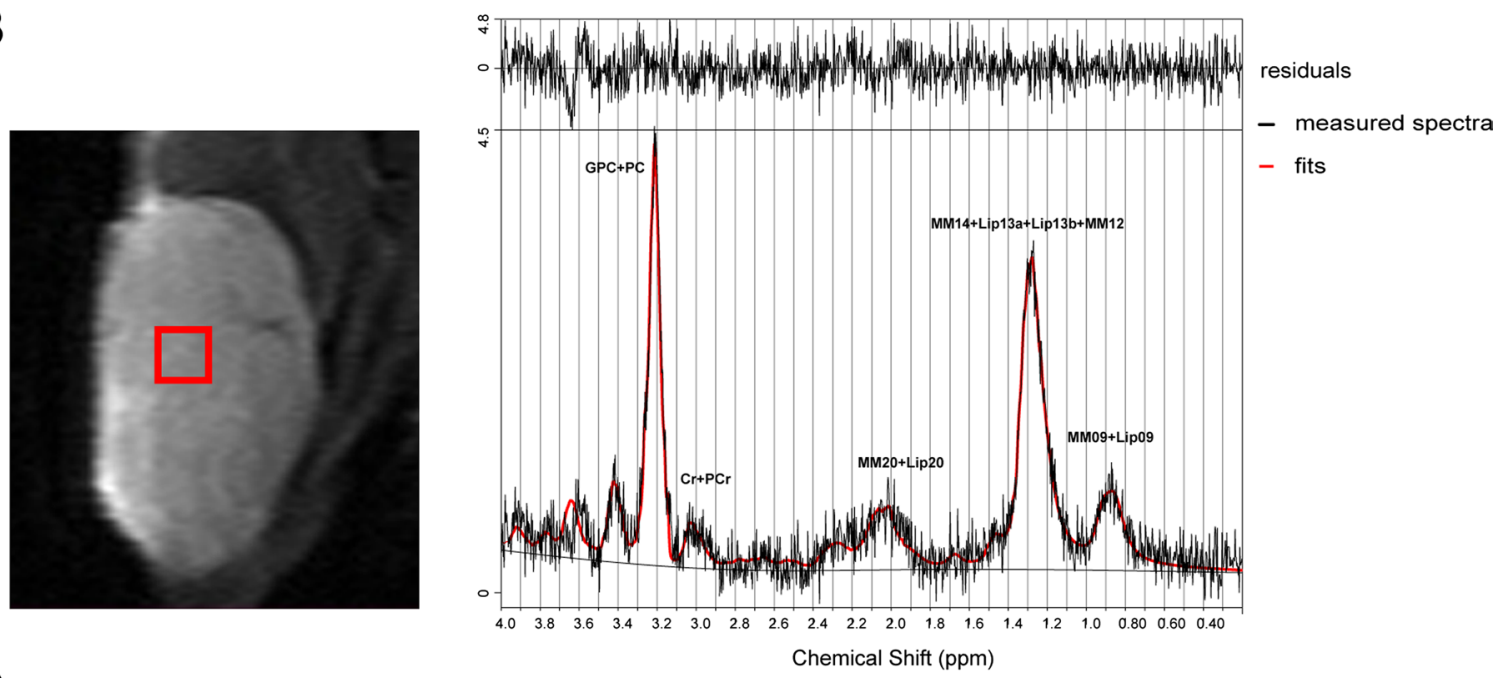

C

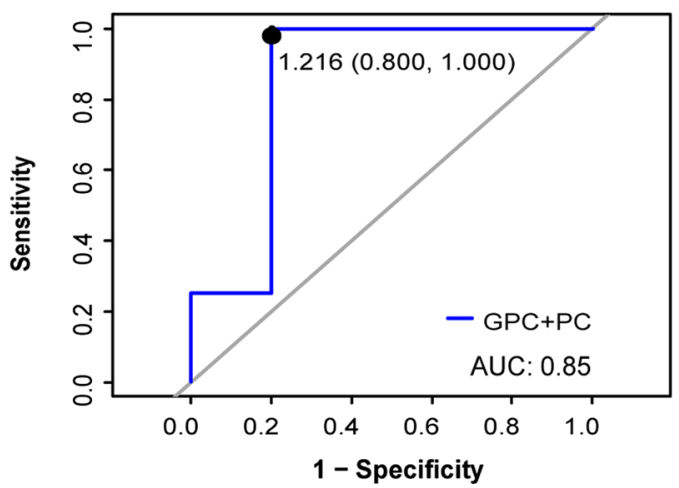

Fig. 3 (See legend on previous page.) 


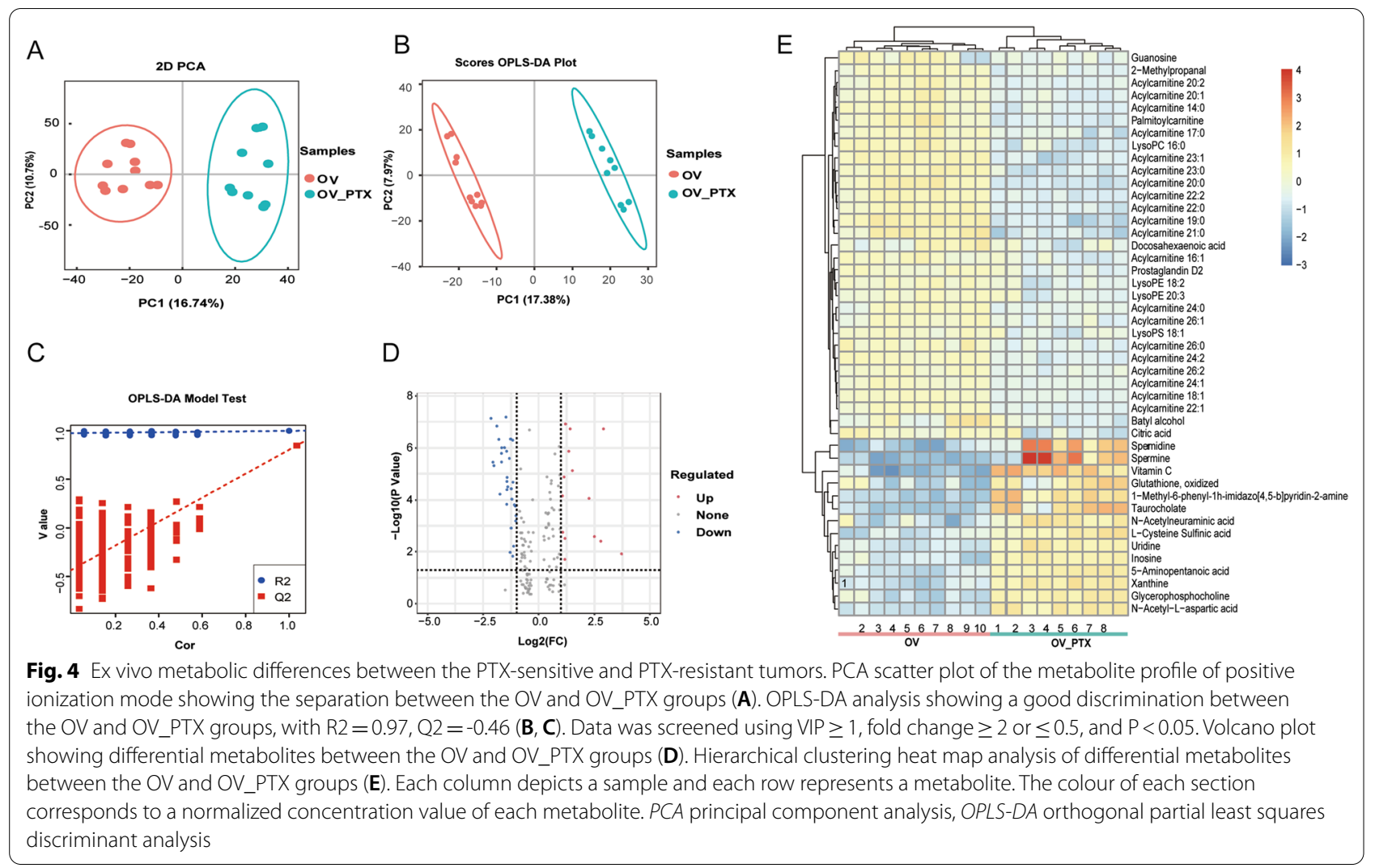

Fig. S1A). Next, the OPLS-DA model was used to further analyze the compounds responsible for the differences between the two groups. Goodness of fit values and predictive ability values indicated that the model possessed a satisfactory fit with a good predictive power (Fig. 4B, C; Additional file 3: Fig. S1B, C). Differential metabolites were screened using VIP and fold change as thresholds. Metabolites that met VIP $\geq 1$, fold change $\geq 2$ or $\leq 0.5$, and $P<0.05$ were considered to be differential metabolites (Fig. 4D, E). Forty-five ex vivo metabolites were identified to be significantly different between the OV tumors and the OV_PTX tumors, with the majority involving of lipids and lipid-like molecules (66.67\%, Fig. 5A, Additional file 4: Table S1). Compared to those in the OV tumors, the levels of 14 metabolites increased while 31 ones decreased in the OV_PTX tumors. Metabolomics analysis showed the top ten differential metabolites according to VIP values: spermine, taurocholate, spermidine, vitamin $\mathrm{C}$, 1-methyl-6-phenyl-1H-imidazo[4,5-b] pyridin-2-amine, acylcarnitine 19:0, glycerophosphocholine, acylcarnitine 14:0, acylcarnitine 17:0, and palmitoylcarnitine (Fig. 5B). Those may be potential biomarkers to identify the PTX-resistant EOC.

Pathway enrichment analysis was performed to identify dysregulated pathways in PTX-resistant tumors. It showed that the commonly dysregulated pathways included lipid metabolism, glycometabolism, amino acid metabolism, nucleotide metabolism, and energy metabolism (Fig. 5C). The top 3 related metabolism pathways were taurine and hypotaurine metabolism, glutathione metabolism and glycerophospholipid metabolism.

The correlation between in vivo differential metabolite $(\mathrm{GPC}+\mathrm{PC})$ and every ex vivo differential metabolite was investigated by calculating the Spearman's correlation coefficient. There were 30 ex vivo differential metabolites that correlated significantly with the differential metabolite GPC $+\mathrm{PC}(|\mathrm{r}|>0.5, P<0.05)$ observed on in vivo ${ }^{1} \mathrm{H}$-MRS, exhibiting the highest positive correlation with GPC $(r=0.885, P<0.001)$ (Fig. 5D). Overall, these data indicated that abnormal Cho concentrations were detected in the PTX-resistant EOCs because of the dysregulated glycerophospholipid metabolism, especially Cho metabolism.

\section{Metabolic enzyme modulation in PTX-resistant ovarian cancer}

According to the major metabolic reprogramming observed in the PTX-resistant EOCs, some essential enzymes might have modulated. Thus we analyzed the proteomics data to identify potential target proteins. A total of 233 differentially expressed proteins, which met fold change $\geq 2$ or $\leq 0.5$, and $P<0.05$, were detected 


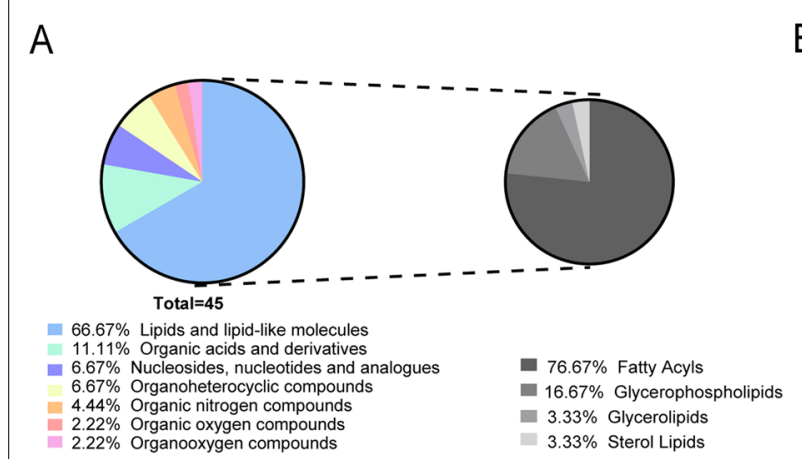

B

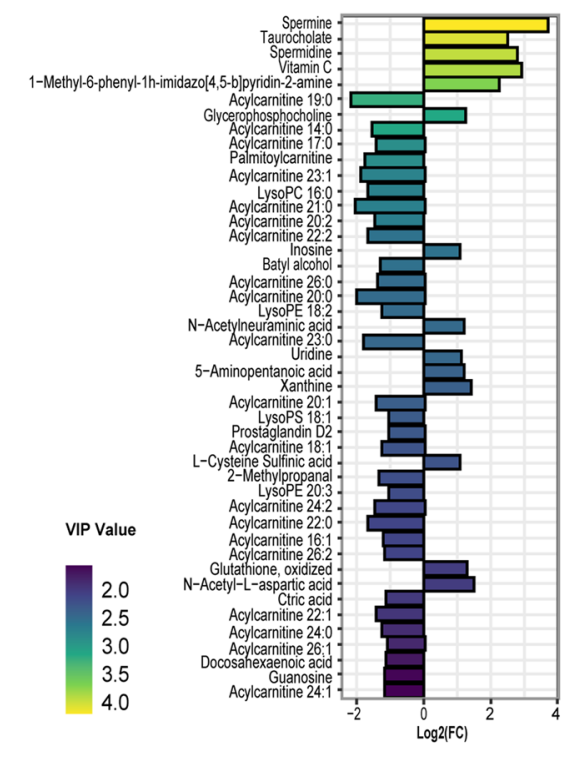

C

D
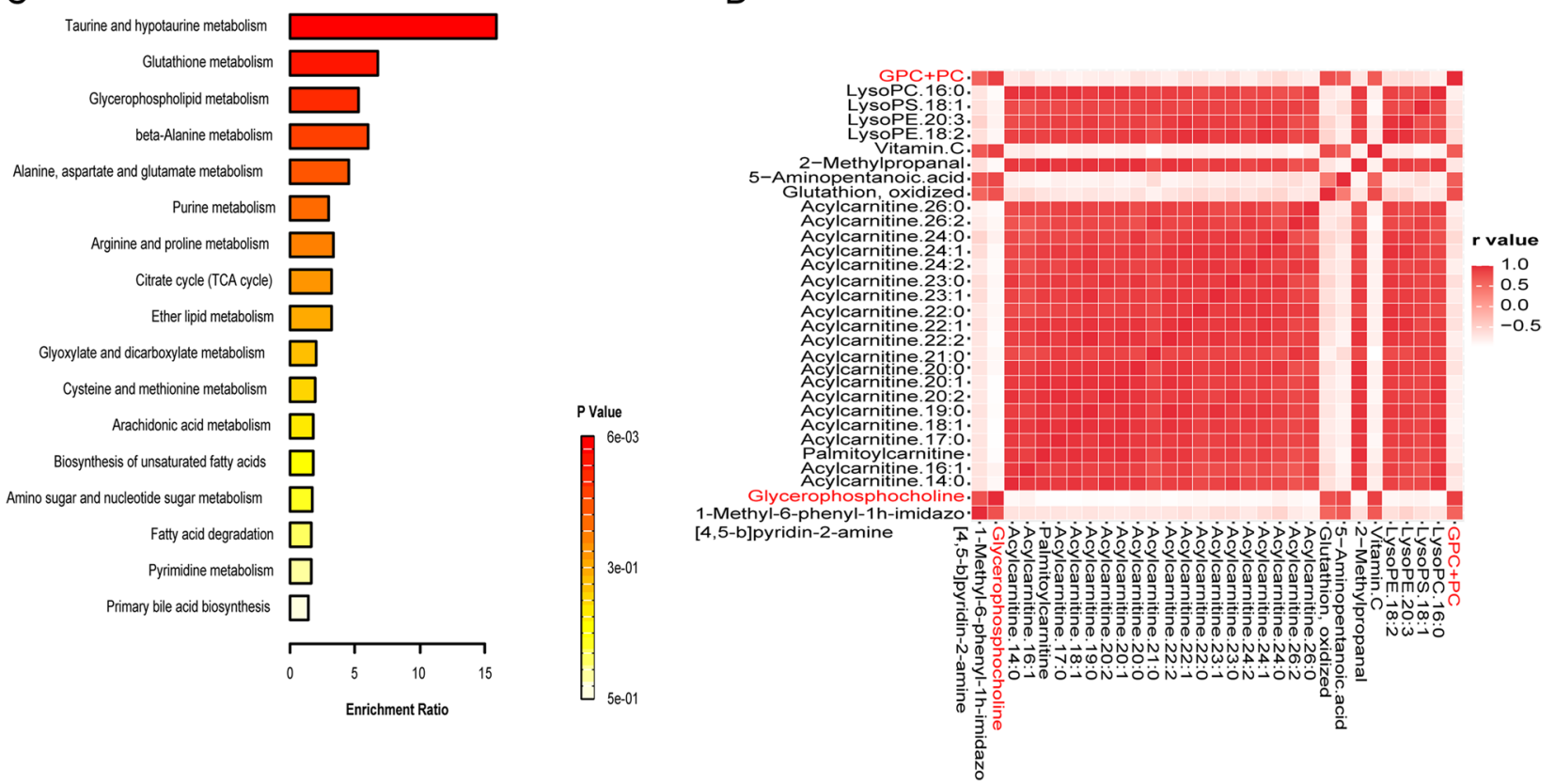

Fig. 5 Ex vivo differential metabolites pathway analysis between the PTX-sensitive and PTX-resistant tumors and the correlation between in vivo and ex vivo differential metabolites. Composition of ex vivo differential metabolites (A). Linear discriminant analysis effect size of ex vivo differential metabolites (B). Pathway enrichment analysis of differential metabolites between the OV and OV_PTX groups (C). Metabolic alterations of the most relevant pathways affected by drug resistance were analyzed by MetaboAnalyst 3.0 databases. Correlation heat map of differential metabolites between in vivo and ex vivo metabolite profiles (D). Each square represents a metabolite and the colour corresponds to its correlation coefficient value compared with other metabolites

between the OV and OV_PTX tumors, without any enzyme of the Cho metabolism (Fig. 6A). GPCPD1 and GDE1 which are responsible for the cleavage of GPC [27] (Fig. 6B) were detectable in the OV tumors, but not in the OV_PTX tumors. Undetectable data in the OV_PTX tumors represented the proteins undetected in over half of the tumors under this experimental methods and conditions. In view of this, we tried to identify GPCPD1and GDE1 since little is known about them in the PTXresistant EOCs. The expressions of GPCPD1 and GDE1 mRNA were significantly lower in the PTX_OV tumors compared to the OV tumors (both $P<0.01$ ) (Fig. 6C).

Next, to address the question that whether gene expression levels of GPCPD1 and GDE1 were related 
A

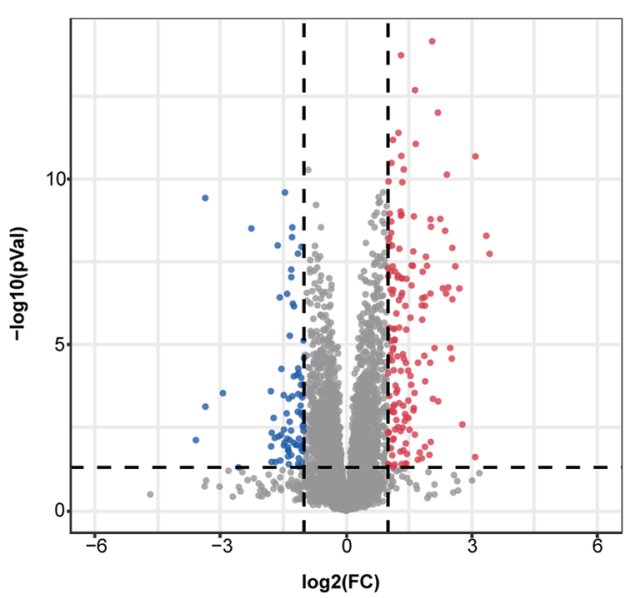

Significant

- Up

- NoDiff

- Down
C

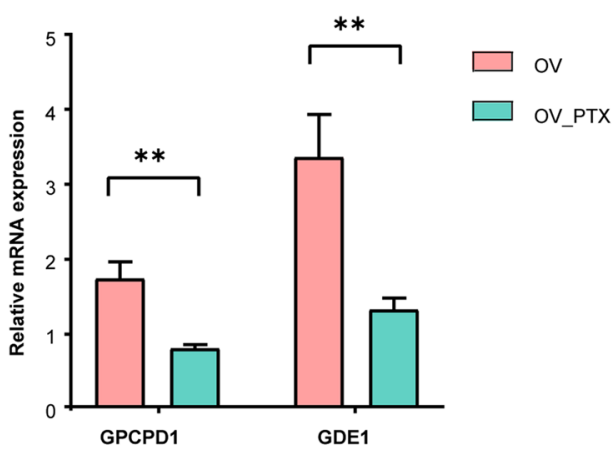

B

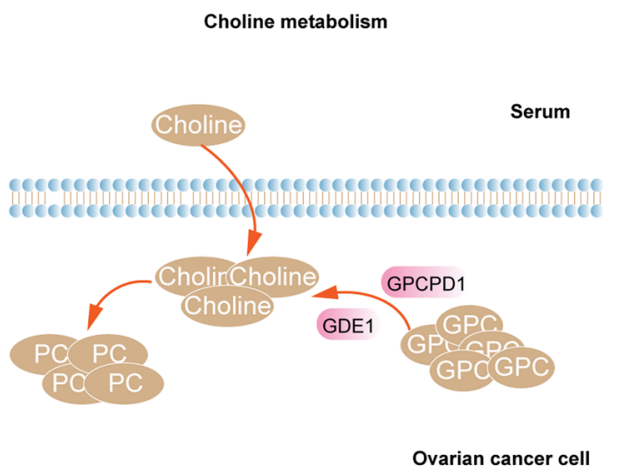

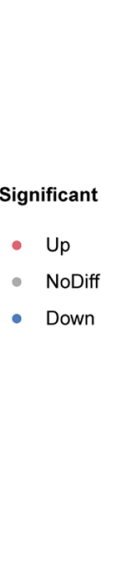

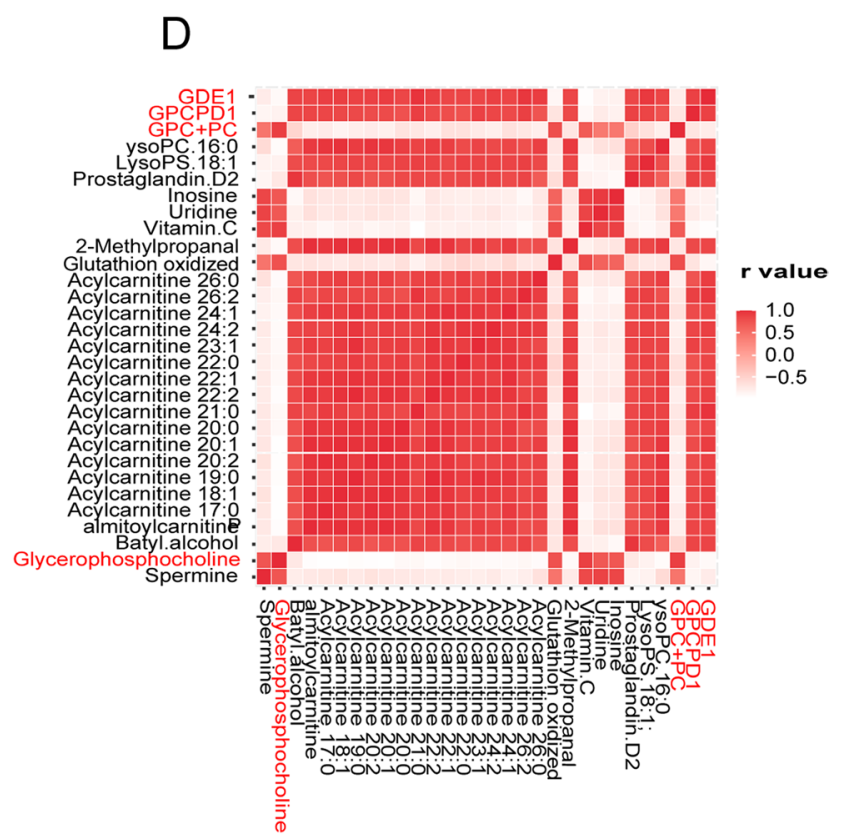

Fig. 6 Regulation of GPCPD1 and GDE1 on PTX-resistance in ovarian cancers. Volcano plot showing differential expressed proteins between the OV and OV_PTX groups (A). Schematic description of Cho metabolism (B). The qRT-PCR showing the expression of GPCPD1 and GDE1 in the OV and OV_PTX groups $(\mathbf{C}) .{ }^{* *}, P<0.01$. Correlation heat map of differential metabolites between GPCPD1 and GDE1 mRNA, in vivo and ex vivo differential metabolites (D). GPCPD1 glycerophosphocholine phosphodiesterase 1, GDE1 glycerophosphodiester phosphodiesterase 1

to metabolic outcome, Spearman correlation coefficients were calculated between protein expressions and differential metabolite levels. GPC $+\mathrm{PC}$, the differential metabolite observed on in vivo ${ }^{1} \mathrm{H}$-MRS, correlated negatively with the mRNA levels of GPCPD1 and GDE1, with $\mathrm{r}=-0.698(P=0.01)$ and $-0.775(P=0.003)$. There were 18 and 26 ex vivo differential metabolites correlated significantly with mRNA levels of GPCPD1 and GDE1 $(|\mathrm{r}|>0.8, P<0.05)$, with a closely negative correlation with GPC ( $\mathrm{r}=-0.841$ and -0.912 , both $P<0.001$, respectively) (Fig. 6D). Expressions of enzymes responsible for GPC decomposition reduced in the OV_PTX tumors, leading to the accumulation of GPC.

\section{Discussion}

Our study introduced the application of in vivo ${ }^{1} \mathrm{H}$-MRS in monitoring the PTX resistance of EOC and indicated that glycerophospholipid metabolism might reprogram 
in the PTX-resistant ovarian cancers. The increased metabolite GPC + PC, namely, Cho, could be detected by in vivo ${ }^{1} \mathrm{H}-\mathrm{MRS}$. Major contributions to GPC accumulation may derive from changes in the activities of enzymes (GPCPD1 and GDE1) involved in Cho metabolism, which is part of glycerophospholipid metabolism.

Metabolic regulation does occur in the PTX-resistant tumors. However, few metabolic studies about drug resistance have been reported. Changes in glycerophospholipid metabolism, sphingolipid metabolism and citric acid metabolism have been found in multidrug-resistant colorectal cancer cell lines using metabolomics analysis [28]. In this study, 45 differential metabolites between the OV and OV-PTX tumors were identified by metabolomics analysis, indicating that glucose metabolism, lipid metabolism, amino acid metabolism, energy metabolism, and nucleotide metabolism (purine, pyrimidine metabolism) had been altered. Pathway enrichment analysis further revealed that lipids and lipid-like molecules of lipid metabolism were predominant ex vivo metabolites. Our results were consistent with those reported by Braun et al. In their study, upregulated aerobic glycolysis and activation of pyrimidine synthesis were found in nab-PTXresistant pancreatic ductal adenocarcinoma cell lines [29].

The PTX-resistant tumors have the altered Cho metabolism. Cho is a major component of glycerophospholipid, and Cho metabolism is an indispensable component of lipid synthesis $[5,30]$. Abnormal Cho metabolism has emerged as a hallmark indicator of cancer [31]. Metabolome has revealed that GPC, PC, Cho and Cho complexes are significantly increased in ovarian cancer tissues [3234]. The tCho level detected by ${ }^{1} \mathrm{H}-\mathrm{MRS}$ has been evaluated as a diagnostic and prognostic biomarker in cancers [13]. In the differential diagnosis of benign and malignant ovarian tumors, a markedly increased Cho level on ${ }^{1} \mathrm{H}$-MRS is considered to be a characteristic of ovarian cancers [14-17]. Our study showed that there was a significant correlation between ex vivo differential metabolites and GPC $+\mathrm{PC}$ on in vivo ${ }^{1} \mathrm{H}$-MRS. GPC was the most significantly upregulated ex vivo metabolite, which indicated that in vivo and ex vivo metabolic characteristics were highly consistent. This observation further suggested that the glycerophospholipids metabolism, especially the Cho metabolism, had changed in the PTXresistant ovarian cancers. Thus, increased GPC + PC levels observed on ${ }^{1} \mathrm{H}$-MRS in our study which represented tCho, might be the result of Cho metabolism reprogramming in the PTX-resistant ovarian cancers.

The tCho on ${ }^{1} \mathrm{H}$-MRS can monitor PTX resistance of tumors. According to the "Response Evaluation Criteria in Solid Tumors" (RECIST), the tumor size change measured by imaging may require three cycles of chemotherapy to determine therapeutic efficacy
[35]. It is reported that patients who showed a greater reduction in tCho than in tumor size are more likely to achieve pathologic complete response [18]. Our ${ }^{1} \mathrm{H}$ MRS findings are accorded with results reported by other researchers that changes of the tCho peak area or height could be used as a biomarker for the therapeutic effect of breast cancer, glioma and liver cancer $[18,22,36]$. A study by Kuo et al. showed that the mean ratios of Cho/lipid were significantly decreased after transcatheter arterial chemoembolization in patients with liver cancer [36]. Another study showed that after treatment with temozolomide for 12 months in patients with low-grade glioma, the tCho was significantly lower than before treatment and after 3 months of treatment [22]. In addition, the Cho/water ratios decreased in accordance with the changes in tumor volume, which suggested that the ratios could reflect the therapeutic effect of temozolomide for glioma [22]. Our in vivo ${ }^{1} \mathrm{H}$-MRS study revealed that GPC + PC (Cho) was significantly higher in the OV_PTX tumors than in OV tumors. Thus, our findings supported that ${ }^{1} \mathrm{H}$-MRS could be used for monitoring PTX-resistant EOCs to allow for early or timely treatment modification.

In addition, our proteomics analysis revealed cholinemetabolizing enzymes were low expression in the PTXresistant EOCs. It is known that GPCPD1 and GDE1 work in a complex enzyme network that regulates Cho metabolism. GPCPD1 cleaves GPC to form glycerol3-phosphate and Cho [27]. GDE1 catalyzes the hydrolysis of various glycerophosphodiesters (including GPC) and releases sn-glycerol 3-phosphate and the corresponding alcohol [37]. Silencing or upregulating these two enzymes can alter GPC, PC and Cho levels [27]. Homozygous deletion of GDE1 results in a buildup of intracellular GPC that is restored to wild-type levels by reintegrating GDE1 into the genome [38]. In Shen's transcriptomics analysis of colon adenocarcinoma, the expression of GDE1 is significantly lower than that of normal tissues, indicating abnormal Cho metabolism in malignant tissues [39]. GPCPD1 has been identified as a key enzyme in the Cho and phospholipid metabolism, which is involved in cell proliferation, migration, invasion, adhesion and spreading $[27,40,41]$. It was reported that doxorubicin decreased the expression of GPCPD1, leading to an ex vivo GPC increase in breast cancer cells [42]. Our correlation analysis indicated that the in vivo metabolites and most of the ex vivo metabolites were related to the expressions of GPCPD1 and GDE1. The proteomics analysis showed GPCPD1 and GDE1 expressions were downregulated in the PTX-resistant EOC and confirmed by a qRT-PCR, resulting in a GPC accumulation and an elevated GPC + PC (Cho) peak on ${ }^{1} \mathrm{H}$-MRS. The different expressions of Cho-metabolizing enzymes between the 
PTX-sensitive and PTX-resistant tumors led to different changes in the GPC, PC and tCho levels, which made GPCPD1 and GDE1 as the potential therapeutic targets.

Our study has some limitations. First, subcutaneous xenograft ovarian cancer models were used in our study, which might differ from orthotopic implantation models due to different biological microenvironments. However, ${ }^{1} \mathrm{H}-$ MRS might be difficult to perform in orthotopic ovarian tumors of nude mice due to small tumor sizes and respiratory movements. Second, compared with metabolomics, lipidomics might cover and detect more lipid and lipidlike metabolites, despite the metabolomics results fully explained the ${ }^{1} \mathrm{H}-\mathrm{MRS}$ findings. Third, Western blot assays were not performed to confirm the protein expression of key enzymes because of limited tumor tissue samples.

In conclusion, our study indicated that the choline metabolic adaptations were associated with the PTX resistance of EOCs. Decreased expressions of GPCPD1 and GDE1 led to increased GPC levels in the PTXresistant EOCs, which could be observed as the tCho on in vivo ${ }^{1} \mathrm{H}$-MRS. These findings suggested that the tCho on in vivo ${ }^{1} \mathrm{H}$-MRS could be used as an indicator for PTX resistance in EOCs.

\begin{abstract}
Abbreviations
AUC: Area under the curve; Ala: Alanine; Asp: Aspartate; Cr: Creatine; EOC: Epithelial ovarian cancer; Ins: Inositol; PTX: Paclitaxel; tCho: Total choline; GDE1: Glycerophosphodiester phosphodiesterase 1; GPC: Glycerophosphocholine; GPCPD1: Glycerophosphocholine phosphodiesterase 1; Lac: Lactate; MM: Macromolecules; Lip: Lipid; NAA: N-acetylaspartate; NAAG: N-acetylaspartylglutamate; OV: OVCAR-3; OV_PTX: Paclitaxel-resistant OVCAR-3; OPLSDA: Orthogonal partial least squares discriminant analysis; PCA: Principal component analysis; PC: Phosphocholine; PCr: Phosphocreatine; Tau: Taurine; VIP: Variable importance in projection; ${ }^{1} \mathrm{H}$-MRS: Proton magnetic resonance spectroscopy.
\end{abstract}

\section{Supplementary Information}

The online version contains supplementary material available at https://doi. org/10.1186/s12967-022-03292-z.

Additional file 1. Supplementary of metabolomics analysis method.

Additional file 2. Supplementary of proteomics analysis method.

Additional file 3: Figure S1. PCA scatter plot of the metabolite profile of negative ionization mode between the PTX-sensitive and PTX-resistant tumors. The OV_PTX group was separated from the OV group (A). OPLSDA analysis showed a good discrimination between the OV and OV_PTX groups, R2 $=0.94, \mathrm{Q} 2=-0.49(\mathrm{~B}, \mathrm{C})$.

Additional file 4: Table S1. Differential metabolites between OV and OV_PTX groups.

\section{Acknowledgements}

We would like to thank Professor Guoxiong Xu for donating paclitaxel-resistant ovarian cancer cells.

\section{Authors' contributions}

All listed authors contributed to the conception, design, operation of experiments, analysis of interpretation of this study's data. JL contributed to study design, analysis, operation of experiements, interpretation, and primary manuscript writing. YL contributed to conception, design, analysis of data. YAL contributed to data collection and formal statistical analysis. LW contributed to operation of experiments and histopathologic analysis. ARZ and XLM contributed to imaging analysis and operation of experiments. JWQ contributed to study conception and design, study analysis and interpretation, and manuscript revisions and approval. All authors read and approved the final manuscript.

\section{Funding}

This work was supported by the National Natural Science Foundation of P.R. China [No.81971579], Shanghai Municipal Commission of Science and Technology [No. 19411972000$]$, Shanghai Municipal Health Commission [No. ZK2019B01] and Shanghai Jinshan District Health Commission [No. JSYQ201901].

\section{Availability of data and materials}

The datasets used and/or analysed during the current study are available from the corresponding author on reasonable request.

\section{Declarations}

\section{Ethics approval and consent to participate}

This study was carried out after the approval of the ethics committee of Shanghai Municipal Public Health Clinical Center (No. 2020-A019-01).

\section{Consent for publication}

Not applicable.

\section{Competing interests}

The authors declare no potential conflicts of interest.

\section{Author details}

'Department of Radiology, Jinshan Hospital, Fudan University, 1508 Longhang Road, Shanghai 201508, People's Republic of China. ${ }^{2}$ Department of Pathology, Jinshan Hospital, Fudan University, 1508 Longhang Road, Shanghai 201508, People's Republic of China.

Received: 19 September 2021 Accepted: 2 February 2022 Published online: 15 February 2022

\section{References}

1. Lheureux S, Gourley C, Vergote I, Vergote I, Oza AM. Epithelial ovarian cancer. Lancet. 2019;393(10177):1240-53. https://doi.org/10.1016/S01406736(18)32552-2

2. Jiang JH, Wang SH, Wang ZH, Cai J, Han LL, Xie LS, et al. HOTAIR promotes paclitaxel resistance by regulating CHEK1 in ovarian cancer. Cancer Chemoth Pharm. 2020;86(2):295-305. https://doi.org/10.1007/ s00280-020-04120-1.

3. Zhang JG, Guan WC, Xu XL, Wang FC, Li X, Xu GX. A novel homeostatic loop of sorcin drives paclitaxel-resistance and malignant progression via Smad4/ZEB1/miR-142-5p in human ovarian cancer. Oncogene. 2021;40(30):4906-18. https://doi.org/10.1038/s41388-021-01891-6.

4. Xia B, Zhao ZT, Wu YY, Wang Y, Zhao Y, Wang J. Circular RNA circTNPO3 regulates paclitaxel resistance of ovarian cancer cells by miR-1299/NEK2 signaling pathway. Mol Ther Nucleic Acids. 2020;21:780-91. https://doi. org/10.1016/j.omtn.2020.06.002

5. Xiong J, Wang L, Fei XC, Jiang XF, Zheng Z, Zhao Y, et al. MYC is a positive regulator of choline metabolism and impedes mitophagy-dependent necroptosis in diffuse large B-cell lymphoma. Blood Cancer J. 2017;7(7): e0. https://doi.org/10.1038/bcj.2017.61.

6. Benjamin DI, Cravatt BF, Nomura DK. Global profiling strategies for mapping dysregulated metabolic pathways in cancer. Cell Metab. 2012;16(5):565-77. https://doi.org/10.1016/j.cmet.2012.09.013.

7. Liu HL, Liu Y, Zhang JT. A new mechanism of drug resistance in breast cancer cells: fatty acid synthase overexpression-mediated palmitate overproduction. Mol Cancer Ther. 2008;7(2):263-70. https://doi.org/10. 1158/1535-7163.MCT-07-0445. 
8. Zhao Y, Butler EB, Tan M. Targeting cellular metabolism to improve cancer therapeutics. Cell Death Dis. 2013;4(3): e532. https://doi.org/10.1038/ cddis.2013.60.

9. Zheng WJ, Feng Q, Liu J, Guo YK, Gao LF, Li RM, et al. Inhibition of 6-phosphogluconate dehydrogenase reverses cisplatin resistance in ovarian and lung cancer. Front Pharmacol. 2017;8:421. https://doi.org/10.3389/fphar. 2017.00421.

10. Zhou M, Zhao YH, Ding Y, Liu H, Liu ZX, Fodstad O, et al. Warburg effect in chemosensitivity: targeting lactate dehydrogenase-A re-sensitizes taxolresistant cancer cells to taxol. Mol Cancer. 2010;9:33. https://doi.org/10. 1186/1476-4598-9-33.

11. Blomme A, Ford CA, Mui E, Patel R, Ntala C, Jamieson LE, et al. 2,4-dienoyl-CoA reductase regulates lipid homeostasis in treatment-resistant prostate cancer. Nat Commun. 2020;11(1):2508. https://doi.org/10.1038/ s41467-020-16126-7.

12. Trousil S, Lee P, Pinato DJ, Ellis JK, Dina R, Aboagye EO, et al. Alterations of choline phospholipid metabolism in endometrial cancer are caused by choline kinase alpha overexpression and a hyperactivated deacylation pathway. Cancer Res. 2014;74(23):6867-77. https://doi.org/10.1158/00085472.CAN-13-2409.

13. Glunde K, Penet M-F, Jiang L, Jacobs MA, Bhujwalla ZM. Choline metabolism-based molecular diagnosis of cancer: an update. Expert Rev Mol Diagn. 2015;15(6):735-47. https://doi.org/10.1586/14737159.2015. 1039515.

14. Mansour SM, Gomma M, Shafik PN. Proton MR spectroscopy and the detection of malignancy in ovarian masses. Br J Radiol. 2019;92(1101):20190134. https://doi.org/10.1259/bjr.20190134.

15. Ma FH, Li YA, Liu J, Li HM, Zhang GF, Qiang JW. Role of proton MR spectroscopy in the differentiation of borderline from malignant epithelial ovarian tumors: a preliminary study. J Magn Reson Imaging. 2019;49(6):1684-93. https://doi.org/10.1002/jmri.26541.

16. Ma FH, Qiang JW, Cai SQ, Zhao SH, Zhang GF, Rao YM. MR spectroscopy for differentiating benign from malignant solid adnexal tumors. AJR Am J Roentgenol. 2015;204(6):W724-30

17. Esseridou A, Leo GD, Sconfienza LM, Caldiera V, Raspagliesi F, Grijuela B, et al. In vivo detection of choline in ovarian tumors using 3D magnetic resonance spectroscopy. Invest Radiol. 2011;46(6):377-82. https://doi. org/10.1097/RLI.0b013e31821690ef.

18. Baek HM, Chen JH, Nie K, Yu HJ, Bahri S, Mehta RS, et al. Predicting pathologic response to neoadjuvant chemotherapy in breast cancer by using MR imaging and quantitative $1 \mathrm{H}$ MR spectroscopy. Radiology. 2009:251(3):653-62. https://doi.org/10.1148/radiol.2512080553.

19. Payne GS, Leach MO. Applications of magnetic resonance spectroscopy in radiotherapy treatment planning. Br J Radiol. 2006;79(Spec No 1):S16-26. https://doi.org/10.1259/bjr/84072695.

20. Meisamy S, Bolan PJ, Baker EH, Bliss RL, Gulbahce E, Everson LI, et al. Neoadjuvant chemotherapy of locally advanced breast cancer: predicting response with in vivo (1)H MR spectroscopy-a pilot study at 4T. Radiology. 2004;233(2):424-31. https://doi.org/10.1148/radiol.2332031285.

21. Guillevin R, Menuel C, Taillibert S, Capelle L, Costalat R, Abud L, et al. Predicting the outcome of grade II glioma treated with temozolomide using proton magnetic resonance spectroscopy. $\mathrm{Br} J \mathrm{Cancer}$. 2011:104(12):1854-61. https://doi.org/10.1038/bjc.2011.174.

22. Murphy PS, Viviers L, Abson C, Rowland IJ, Brada M, Leach MO, et al. Monitoring temozolomide treatment of low-grade glioma with proton magnetic resonance spectroscopy. Br J Cancer. 2004;90(4):781-6. https:// doi.org/10.1038/sj.bjc.6601593.

23. Wang FC, Zhang LY, Liu J. Highly expressed STAT1 contributes to the suppression of stemness properties in human paclitaxel-resistant ovarian cancer cells. Aging (Albany NY). 2020;12(11):11042-60. https://doi.org/10. 18632/aging. 103317.

24. Tomayko MM, Reynolds CP. Determination of subcutaneous tumor size in athymic (nude) mice. Cancer Chemother Pharmacol. 1989;24(3):148-54. https://doi.org/10.1007/BF00300234.

25. Provencher SW. Estimation of metabolite concentrations from localized in vivo proton NMR spectra. Magn Reson Med. 1993;30(6):672-9. https:// doi.org/10.1002/mrm.1910300604.

26. Terpstra M, Cheong I, Lyu T, Deelchand DK, Emir UE, Bednarik P, et al. Test-retest reproducibility of neurochemical profifiles with short-echo, single-voxel MR spectroscopy at 3T and 7T. Magn Reson Med. 2016;76(4):1083-91. https://doi.org/10.1002/mrm.26022.

27. Stewart JD, Marchan R, Lesjak MS, Lambert J, Hergenroeder R, Ellis JK, et al. Choline-releasing glycerophosphodiesterase EDI3 drives tumor cell migration and metastasis. Proc Natl Acad Sci U S A. 2012;109(21):8155-60. https://doi.org/10.1073/pnas.1117654109.

28. Wang XY, Wang ZH, Wang KL, Gao M, Zhang H, Xu X. Metabolomics analysis of multidrug resistance in colorectal cancer cell and multidrug resistance reversal effect of verapamil. Biomed Chromatogr. 2021;35(2): e4976. https://doi.org/10.1002/bmc.4976.

29. Braun LM, Lagies S, Guenzle J, Fichtner-Feigl S, Wittel UA, Kammerer B. Metabolic adaptation during nab-paclitaxel resistance in pancreatic cancer cell lines. Cells. 2020;9(5):1251. https://doi.org/10.3390/cells9051251.

30. Xiong J, Bian J, Wang L, Zhou JY, Wang Y, Zhao Y, et al. Dysregulated choline metabolism in T-cell lymphoma: role of choline kinase-alpha and therapeutic targeting. Blood Cancer J. 2015;5(3):287. https://doi.org/10. 1038/bcj.2015.10

31. Glunde K, Bhujwalla ZM, Ronen SM. Choline metabolism in malignant transformation. Nat Rev Cancer. 2011;11(12):835-48. https://doi.org/10. 1038/nrc3162.

32. Sellem DB, Elbayed K, Neuville A, Moussallieh FM, Lang-Averous G, Piotto $M$, et al. Metabolomic characterization of ovarian epithelial carcinomas by HRMAS-NMR spectroscopy. J Oncol. 2011;2011: 174019. https://doi.org/ 10.1155/2011/174019.

33. Boss EA, Moolenaar SH, Massuger LF, Boonstra H, Engelke UF, de Jong JG, et al. High-resolution proton nuclear magnetic resonance spectroscopy of ovarian cyst fluid. NMR Biomed. 2000;13(5):297-305. https://doi.org/10. 1002/1099-1492(200008)13:5\%3c297::aid-nbm648\%3e3.0.co;2-i.

34. Iorio E, Mezzanzanica D, Alberti P, Spadaro F, Ramoni C, D'Ascenzo S, et al. Alterations of choline phospholipid metabolism in ovarian tumor progression. Cancer Res. 2005;65(20):9369-76. https://doi.org/10.1158/ 0008-5472.CAN-05-1146.

35. Eisenhauer EA, Therasse P, Bogaerts J, Schwartz LH, Sargent D, Ford R, et al. New response evaluation criteria in solid tumours: revised RECIST guideline (version 1.1). Eur J Cancer. 2009;45(2):228-47. https://doi.org/ 10.1016/j.ejca.2008.10.026.

36. Kuo YT, Li CW, Chen CY, Jao J, Wu DK, Liu GC. In vivo proton magnetic resonance spectroscopy of large focal hepatic lesions and metabolite change of hepatocellular carcinoma before and after transcatheter arterial chemoembolization using 3.0-T MR scanner. J Magn Reson Imaging. 2004;19(5):598-604. https://doi.org/10.1002/jmri.20046.

37. Der Rest BV, Rolland N, Boisson A-M, Ferro M, Bligny R, Douce R. Identification and characterization of plant glycerophosphodiester phosphodiesterase. Biochem J. 2004;379(Pt3):601-7. https://doi.org/10.1042/BJ200 31489.

38. Bishop AC, Ganguly S, Solis NV, Cooley BM, Jensen-Seaman MI, Filler SG, et al. Glycerophosphocholine utilization by Candida albicans: role of the Git3 transporter in virulence. J Biol Chem. 2013;288(47):33939-52. https:// doi.org/10.1074/jbc.M113.505735.

39. Shen Q, Lu C, Yang H, Ge MX, Xia WX, Kong QP, et al. Glycerophosphodiester phosphodiesterase 1 (GDE1) acts as a potential tumor suppressor and is a novel therapeutic target for non-mucin-producing colon adenocarcinoma. PeerJ. 2020;8: e8421. https://doi.org/10.7717/peeri.8421.

40. Lesjak MS, Marchan R, Stewart JD, Rempel E, Rahnenführer J, Hengstler JG. EDI3 links choline metabolism to integrin expression, cell adhesion and spreading. Cell Adh Migr. 2014;8(5):499-508. https://doi.org/10.4161/ cam.29284.

41. Marchan R, Lesjak MS, Stewart JD, Winter R, Seeliger J, Hengstler JG. Choline-releasing glycerophosphodiesterase EDI3 links the tumor metabolome to signaling network activities. Cell Cycle. 2012;11(24):4499-506. https://doi.org/10.4161/cc.22544.

42. Cheng ML, Rizwan A, Jiang L, Bhujwalla ZM, Glunde K. Molecular effects of doxorubicin on choline metabolism in breast cancer. Neoplasia. 2017;19(8):617-27. https://doi.org/10.1016/j.neo.2017.05.004.

\section{Publisher's Note}

Springer Nature remains neutral with regard to jurisdictional claims in published maps and institutional affiliations. 